\title{
MONODROMY GROUP FOR A STRONGLY SEMISTABLE PRINCIPAL BUNDLE OVER A CURVE, II
}

\author{
INDRANIL BISWAS AND A. J. PARAMESWARAN
}

\begin{abstract}
Let $X$ be a geometrically irreducible smooth projective curve defined over a field $k$. Assume that $X$ has a $k$-rational point; fix a $k$-rational point $x \in X$. From these data we construct an affine group scheme $\mathcal{G}_{X}$ defined over the field $k$ as well as a principal $\mathcal{G}_{X}$-bundle $E_{\mathcal{G}_{X}}$ over the curve $X$. The group scheme $\mathcal{G}_{X}$ is given by a $\mathbb{Q}$-graded neutral Tannakian category built out of all strongly semistable vector bundles over $X$. The principal bundle $E_{\mathcal{G}_{X}}$ is tautological. Let $G$ be a linear algebraic group, defined over $k$, that does not admit any nontrivial character which is trivial on the connected component, containing the identity element, of the reduced center of $G$. Let $E_{G}$ be a strongly semistable principal $G$-bundle over $X$. We associate to $E_{G}$ a group scheme $M$ defined over $k$, which we call the monodromy group scheme of $E_{G}$, and a principal $M$-bundle $E_{M}$ over $X$, which we call the monodromy bundle of $E_{G}$. The group scheme $M$ is canonically a quotient of $\mathcal{G}_{X}$, and $E_{M}$ is the extension of structure group of $E_{\mathcal{G}_{X}}$. The group scheme $M$ is also canonically embedded in the fiber $\operatorname{Ad}\left(E_{G}\right)_{x}$ over $x$ of the adjoint bundle.
\end{abstract}

\section{INTRODUCTION}

Let $X$ be a geometrically irreducible smooth projective curve defined over a field $k$ such that $X$ admits a $k$-rational point. Fix a $k$-rational point $x$ of $X$. From this data we construct a neutral Tannakian category $\mathcal{C}_{X}$ defined over the field $k$ in the following way. The objects of $\mathcal{C}_{X}$ are all maps $f$ from the rational numbers to the strongly semistable vector bundles over $X$ such that $f(\lambda)=0$ for all but finitely many $\lambda \in \mathbb{Q}$, and if $f(\lambda) \neq 0$, then

$$
\mu(f(\lambda)):=\frac{\operatorname{degree}(f(\lambda))}{\operatorname{rank}(f(\lambda))}=\lambda .
$$

For any $f, f^{\prime} \in \mathcal{C}_{X}$, set

$$
\operatorname{Hom}\left(f, f^{\prime}\right):=\prod_{\lambda \in \mathbb{Q}} H^{0}\left(X, \operatorname{Hom}\left(f(\lambda), f^{\prime}(\lambda)\right)\right) .
$$

We note that for any two vector bundles $V$ and $W$ over $X$, with

$$
\mu(V):=\frac{\operatorname{degree}(V)}{\operatorname{rank}(V)}=\frac{\operatorname{degree}(W)}{\operatorname{rank}(W)}=: \mu(W),
$$

we have $\mu(V \oplus W)=\mu(V)$. If $V$ and $W$ are also strongly semistable, then for any

$$
\phi \in H^{0}(X, \operatorname{Hom}(V, W)),
$$

2000 Mathematics Subject Classification. 14L15, 14L17.

Key words and phrases. Neutral Tannakian category, principal bundle, semistability. 
either the homomorphism $\phi$ is injective (respectively, surjective) or kernel $(\phi)$ (respectively, cokernel $(\phi))$ is a strongly semistable vector bundle with same degree/rank quotient as that of $V$; the details are in Section 2. These properties imply that $\mathcal{C}_{X}$ is an abelian category.

For any $f, f^{\prime} \in \mathcal{C}_{X}$, define their tensor product

$$
\left(f \otimes f^{\prime}\right)(\lambda):=\bigoplus_{z \in \mathbb{Q}} f(z) \otimes f^{\prime}(\lambda-z),
$$

and define $f^{*}$ by $f^{*}(\lambda)=f(-\lambda)^{*}$. It can be shown that $f \otimes f^{\prime}, f^{*} \in \mathcal{C}_{X}$. The object $f_{0} \in \mathcal{C}_{X}$, defined by $f_{0}(\lambda)=0$ for $\lambda \neq 0$ and $f_{0}(0)=\mathcal{O}_{X}$ (the structure sheaf of $X$ ), acts as the identity element for the tensor product operation on $\mathcal{C}_{X}$.

Using the $k$-rational point $x \in X$, we have a fiber functor on $\mathcal{C}_{X}$ that sends any object $f$ to the $k$-vector space

$$
\bigoplus_{z \in \mathbb{Q}} f(z)_{x}
$$

All these operations together define a neutral Tannakian category over $k$. Let $\mathcal{G}_{X}$ denote the affine group scheme defined over $k$ given by this neutral Tannakian category $\mathcal{C}_{X}$.

Let $\operatorname{Vect}(X)$ denote the category of vector bundles over the curve $X$. We have a covariant functor from $\mathcal{C}_{X}$ to $\operatorname{Vect}(X)$ defined by

$$
f \longmapsto \bigoplus_{z \in \mathbb{Q}} f(z)
$$

This functor is compatible with the operations of tensor product, direct sum and dualization. Therefore, this functor defines a principal $\mathcal{G}_{X}$-bundle $E_{\mathcal{G}_{X}}$ over $X$.

The fundamental group scheme of $X$ constructed in [6], [7] is a quotient of $\mathcal{G}_{X}$.

Let $G$ be a linear algebraic group defined over the field $k$ with the property that there is no nontrivial character of $G$ which is trivial on the center of $G$. Let $Z_{0}(G)$ denote the maximal split torus contained in the reduced center of $G$.

Take a strongly semistable principal $G$-bundle $E_{G}$ over $X$. Given a finite dimensional left $G$-module $V$, consider the isotypical decomposition

$$
V=\bigoplus_{\chi \in Z_{0}(G)^{*}} V_{\chi}
$$

of the $Z_{0}(G)$-module $V$, where $Z_{0}(G)^{*}$ is the group of characters of $Z_{0}(G)$. Since the actions of $Z_{0}(G)$ and $G$ on $V$ commute, each $V_{\chi}$ is a $G$-module. Let $E_{V}$ (respectively, $\left.E_{V_{\chi}}\right)$ be the vector bundle over $X$ associated to the principal $G$-bundle $E_{G}$ for the $G$ module $V$ (respectively, $V_{\chi}$ ). It can be shown that the vector bundle $V_{\chi}$ is strongly semistable. Also, we have a homomorphism

$$
\delta_{E_{G}}: Z_{0}(G)^{*} \longrightarrow \mathbb{Q}
$$

that sends any character $\chi^{\prime}$ to $\frac{\operatorname{degree}\left(V_{\chi^{\prime}}^{\prime}\right)}{\operatorname{rank}\left(V_{\chi^{\prime}}^{\prime}\right)}$ for some $G$-module $V^{\prime}$; it does not depend on the choice of $V^{\prime}$ (Corollary 3.3). 
Therefore, for any finite dimensional left $G$-module $V$, we have an object $f_{E_{G}, V}$ of $\mathcal{C}_{X}$ defined by

$$
f_{E_{G}, V}(\lambda):=\bigoplus_{\left\{\chi \in Z_{0}(G)^{*} \mid \delta_{E_{G}}(\chi)=\lambda\right\}} E_{V_{\chi}} .
$$

We construct a subcategory of the Tannakian category $\mathcal{C}_{X}$ by considering all objects of $\mathcal{C}_{X}$ isomorphic to some subquotient of some $f_{E_{G}, V}$, where $V$ runs over all finite dimensional left $G$-modules. This subcategory gives a quotient group scheme $\mathcal{G}_{X}$, which we call the monodromy group scheme of $E_{G}$. Let $M$ denote the monodromy group scheme of $E_{G}$. Let $E_{M}$ be the principal $M$-bundle over $X$ obtained by extending the structure group of the principal $\mathcal{G}_{X}$-bundle $E_{\mathcal{G}_{X}}$. The details of these constructions are in Section 3 ,

In [1], the monodromy group scheme and the monodromy bundle were constructed under the extra assumptions that the base field is algebraically closed and the group $G$ is semisimple.

\section{A universal TANNAKIAN CATEgory For A POINTED CURVE}

Let $k$ be any field. Let $X$ be a geometrically irreducible smooth projective curve defined over $k$.

A vector bundle $W$ over $X$ is called semistable if for every subbundle $W^{\prime} \subset W$ of positive rank, the inequality degree $\left(W^{\prime}\right) / \operatorname{rank}\left(W^{\prime}\right) \leq \operatorname{degree}(W) \operatorname{rank}(W)$ holds. We

recall that the rational number $\frac{\operatorname{degree}(W)}{\operatorname{rank}(W)}$ is called the slope of $W$, and it is denoted by $\mu(W)$.

Proposition 2.1 ([5], [4]). Let $\ell$ be a field extension of $k$. A vector bundle $W$ over $X$ is semistable if and only if the base change $W \bigotimes_{k} \ell$ over $X \times_{k} \ell$ is semistable.

The above proposition is proved in [5] under the assumption that $k$ is infinite (see [5, page 97, Proposition 3]), and it is proved in [4] under the assumption that $k$ is perfect (see [4, page 222]). We note that if $W$ is not semistable, then it is immediate that $W \bigotimes_{k} \ell$ is not semistable.

Consider the diagram

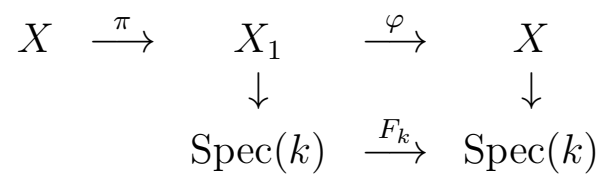

where $F_{k}$ is the Frobenius map of $k$ if the characteristic of the field $k$ is positive, and it is the identity map when the characteristic is zero, the square is Cartesian, and $\pi$ is the relative Frobenius map (see [9, page 118]). The composition $\varphi \circ \pi$ will be denoted by $F_{X}$.

A semistable vector bundle $W$ over $X$ is called strongly semistable if the iterated pull back

$$
(\overbrace{F_{X} \circ \cdots \circ F_{X}}^{n-\text { times }}) * W
$$

is semistable for all $n \geq 1$. 
Remark 2.2. Strongly semistable vector bundle are usually defined under the assumption that the base field is perfect. In view of Proposition[2.1, the above definition is compatible with it.

Let $\mathcal{C}_{X}$ denote the space of all maps $f$ from $\mathbb{Q}$ to the space of all strongly semistable vector bundles over $X$ satisfying the following two conditions:

- All but finitely many rational numbers are sent by $f$ to the vector bundle of rank zero.

- For any $\eta \in \mathbb{Q}$ with $f(\eta) \neq 0$,

$$
\mu(f(\eta)):=\frac{\operatorname{degree}(f(\eta))}{\operatorname{rank}(f(\eta))}=\eta .
$$

Therefore, $\mathcal{C}_{X}$ consists of finite collections of strongly semistable vector bundles over $X$ of distinct slopes. In other words, any element of $\mathcal{C}_{X}$ is of the form

$$
\underline{V}:=\left(V_{\lambda_{1}}, \cdots, V_{\lambda_{n}}\right),
$$

where $\lambda_{1}<\cdots<\lambda_{n}$ are finitely many (possibly empty) rational numbers, and for each $i \in[1, n], V_{i}$ is a strongly semistable vector bundle over $X$ with

$$
\mu\left(V_{i}\right)=\lambda_{i} .
$$

For any $f, f^{\prime} \in \mathcal{C}_{X}$, define $f \bigoplus f^{\prime} \in \mathcal{C}_{X}$ to be the function that sends any $\lambda \in \mathbb{Q}$ to the direct sum of vector bundles $f(\lambda) \bigoplus f^{\prime}(\lambda)$. Since both $f(\lambda)$ and $f^{\prime}(\lambda)$ are strongly semistable with

$$
\mu(f(\lambda))=\mu\left(f^{\prime}(\lambda)\right)=\lambda
$$

provided $f(\lambda) \neq 0 \neq f^{\prime}(\lambda)$, the vector bundle $f(\lambda) \bigoplus f^{\prime}(\lambda)$ is also strongly semistable with

$$
\mu\left(f(\lambda) \oplus f^{\prime}(\lambda)\right)=\lambda
$$

provided $f(\lambda) \bigoplus f^{\prime}(\lambda) \neq 0$.

If $V$ and $W$ are two strongly semistable vector bundles over $X$, then the vector bundle $V \otimes W$ is also strongly semistable; this follows from Remark 2.2] and [8, page 288, Theorem 3.23] (reproduced in Theorem 3.5). We further have

$$
\mu(V \bigotimes W)=\mu(V)+\mu(W)
$$

This enables us to define the tensor product operation on $\mathcal{C}_{X}$ in the following way.

For any $f, f^{\prime} \in \mathcal{C}_{X}$, define

$$
\left(f \otimes f^{\prime}\right)(\lambda):=\bigoplus_{z \in \mathbb{Q}} f(z) \otimes f^{\prime}(\lambda-z) .
$$

If other words, if $f=\underline{V}$ as in eqn. (2.2), then

$$
\left(f \otimes f^{\prime}\right)(\lambda)=\left(V_{\lambda_{1}} \bigotimes f^{\prime}\left(\lambda-\lambda_{1}\right)\right) \bigoplus\left(V_{\lambda_{2}} \bigotimes f^{\prime}\left(\lambda-\lambda_{2}\right)\right) \bigoplus \cdots \bigoplus\left(V_{\lambda_{n}} \bigotimes f^{\prime}\left(\lambda-\lambda_{n}\right)\right) .
$$

Since both $f(c)$ and $f^{\prime}(c)$ are zero except for finitely many $c$, eqn. (2.4) is a finite direct sum. Therefore, using eqn. (2.3) we conclude that $f \otimes f^{\prime} \in \mathcal{C}_{X}$. 
For any $f \in \mathcal{C}_{X}$, define the dual $f^{*}$ of $f$ to be the function from $\mathbb{Q}$ to the strongly semistable vector bundles over $X$ that sends any $\lambda \in \mathbb{Q}$ to the dual vector bundle $f(-\lambda)^{*}$. Clearly, we have $f^{*} \in \mathcal{C}_{X}$.

For any $f, f^{\prime} \in \mathcal{C}_{X}$, a homomorphism from $f$ to $f^{\prime}$ is a function

$$
\gamma: \mathbb{Q} \longrightarrow \bigoplus_{z \in \mathbb{Q}} H^{0}\left(X, \operatorname{Hom}\left(f(z), f^{\prime}(z)\right)\right)
$$

such that $\gamma(z) \in H^{0}\left(X, \operatorname{Hom}\left(f(z), f^{\prime}(z)\right)\right)$ for all $z \in \mathbb{Q}$. By $\operatorname{Hom}\left(f, f^{\prime}\right)$ we will denote the set of all homomorphisms from $f$ to $f^{\prime}$. So

$$
\operatorname{Hom}\left(f, f^{\prime}\right):=\prod_{\lambda \in \mathbb{Q}} H^{0}\left(X, \operatorname{Hom}\left(f(\lambda), f^{\prime}(\lambda)\right)\right) .
$$

A homomorphism $\gamma$ from $f$ to $f^{\prime}$ will be called an isomorphism if

$$
\gamma(z): f(z) \longrightarrow f^{\prime}(z)
$$

is an isomorphism for all $z \in \mathbb{Q}$.

Let $\phi: V \longrightarrow W$ be a homomorphism between strongly semistable vector bundles $V$ and $W$ over $X$ with

$$
\mu(V)=\mu(W) .
$$

Then it can be shown that either $\phi$ is injective, or $\operatorname{kernel}(\phi)$ is a strongly semistable vector bundle over $X$ with

$$
\mu(\operatorname{kernel}(\phi))=\mu(V) .
$$

Indeed, if $\phi$ is nonzero with $\operatorname{kernel}(\phi)$ a nonzero subsheaf of $V$, then consider $V / \operatorname{kernel}(\phi)$. We note that $V / \operatorname{kernel}(\phi)$ is a quotient of $V$ as well as a subsheaf of $W$. Therefore, as $V$ and $W$ are semistable, we have

$$
\mu(V) \leq \mu(V / \operatorname{kernel}(\phi)) \leq \mu(W) .
$$

Now from eqn. (2.5) it follows that both the inequalities in eqn. (2.6) are equalities. Consequently, $\mu(V)=\mu(\operatorname{kernel}(\phi))$. It also follows that $V / \operatorname{kernel}(\phi)$ is torsionfree, because the inverse image in $V$, of the torsion part of $V / \operatorname{kernel}(\phi)$, has slope strictly greater than $\mu((\operatorname{kernel}(\phi))$ if $V / \operatorname{kernel}(\phi)$ has torsion. Note that as $V$ is semistable, it does not have any subsheaf with slope larger than $\mu(V)$. Since $V / \operatorname{kernel}(\phi)$ is torsionfree, we conclude that $\operatorname{kernel}(\phi)$ is a subbundle of $V$.

Similarly, either $\phi$ is surjective or cokernel $(\phi):=W / \phi(V)$ is a strongly semistable vector bundle over $X$ with

$$
\mu(\operatorname{cokernel}(\phi))=\mu(W) .
$$

(Replace $\phi$ by its dual $\phi^{*}$ in the above argument.) This enables us to define the kernel and the cokernel of any homomorphism between two objects in $\mathcal{C}_{X}$.

Take any $f, f^{\prime} \in \mathcal{C}_{X}$ and any $\gamma \in \operatorname{Hom}\left(f, f^{\prime}\right)$. Consider the function from $\mathbb{Q}$ to the space of all strongly semistable vector bundles over $X$ that sends any $\lambda \in \mathbb{Q}$ to the kernel of the homomorphism

$$
\gamma(\lambda): f(\lambda) \longrightarrow f^{\prime}(\lambda)
$$

This function defines an object of $\mathcal{C}_{X}$, which we will call the kernel of $\gamma$, and it will be denoted by $\operatorname{kernel}(\gamma)$. Similarly, consider the function from $\mathbb{Q}$ to the space of all 
strongly semistable vector bundles over $X$ that sends any $\lambda \in \mathbb{Q}$ to the cokernel of the homomorphism

$$
\gamma(\lambda): f(\lambda) \longrightarrow f^{\prime}(\lambda)
$$

The object of $\mathcal{C}_{X}$ defined by this function will be denoted by cokernel $(\gamma)$, and it will be called the cokernel of $\gamma$.

Remark 2.3. The abelian category

$$
\mathcal{C}_{X}=\bigoplus_{\lambda \in \mathbb{Q}} \mathcal{C}_{X}^{\lambda}
$$

where $\mathcal{C}_{X}^{\lambda}$ is the abelian category of strongly semistable vector bundles over $X$ of slope $\lambda$. The index $\mathbb{Q}$ in the above direct sum acts as a weight that guides the tensor product and the dualization operations.

Take any closed point $x$ of $X$. The residue field will be denoted by $k(x)$. For any vector bundle $V$ over $X$, the fiber of $V$ over $x$, which is a $k(x)$-vector space, will be denoted by $V_{x}$. The category of finite dimensional vector spaces over the field $k(x)$ will be denoted by $k(x)$-mod.

We have a functor

$$
\omega: \mathcal{C}_{X} \longrightarrow k(x)-\bmod
$$

defined by

$$
f \longmapsto \bigoplus_{z \in \mathbb{Q}} f(z)_{x}
$$

In other words, for any

$$
\underline{V}:=\left(V_{\lambda_{1}}, \cdots, V_{\lambda_{n}}\right) \in \mathcal{C}_{X}
$$

as in eqn. (2.2), we have $\omega(\underline{V})=\left(V_{\lambda_{1}}\right)_{x} \bigoplus \cdots \bigoplus\left(V_{\lambda_{n}}\right)_{x}$.

Let $\mathcal{O}_{X}$ be the trivial line bundle over $X$ defined by the structure sheaf of $X$. The object in $\mathcal{C}_{X}$ defined by $\mathcal{O}_{X}$ will also be denoted by $\mathcal{O}_{X}$.

The triple $\left(\mathcal{C}_{X}, \mathcal{O}_{X}, \omega\right)$ together form a Tannakian category over $k$; see [10], [3] for Tannakian category. We recall that a Tannakian category over $k$ is a rigid abelian tensor category $\mathcal{C}^{\prime}$ such that

- $\operatorname{End}(\mathbb{1})=k$, and

- there is a field extension $k^{\prime}$ of $k$ and a $k$-linear fiber functor from $\mathcal{C}^{\prime}$ to the category of vector spaces over $k^{\prime}$.

Henceforth, we will assume that $X$ admits a $k$-rational point. Fix a $k$-rational point $x$ of $X$.

Since $k(x)=k$, the above Tannakian category defined by the triple $\left(\mathcal{C}_{X}, \mathcal{O}_{X}, \omega\right)$ is a neutral Tannakian category. Hence they define a affine group scheme defined over $k$, 3 , page 130, Theorem 2.11], [7, Theorem 1.1], [10, Theorem 1].

Let $\mathcal{G}_{X}$ denote the group scheme defined over $k$ given by the neutral Tannakian category $\left(\mathcal{C}_{X}, \mathcal{O}_{X}, \omega\right)$.

We note that the above neutral Tannakian category is $\mathbb{Q}$-graded (see [3, page 186]). We will now show that there is a tautological principal $\mathcal{G}_{X}$-bundle over $X$. 
Take any object

$$
\underline{V}:=\left(V_{\lambda_{1}}, \cdots, V_{\lambda_{n}}\right) \in \mathcal{C}_{X}
$$

as in eqn. (2.2). To it we associate the vector bundle $\left(V_{\lambda_{1}} \bigoplus \cdots \bigoplus V_{\lambda_{n}}\right)$. In other words, we have a functor

$$
\mathcal{C}_{X} \longrightarrow \operatorname{Vect}(X)
$$

where $\operatorname{Vect}(X)$ as before is the category of vector bundles over $X$, defined by

$$
f \longmapsto \bigoplus_{z \in \mathbb{Q}} f(z)
$$

Using [3, page 149, Theorem 3.2], this functor gives a principal $\mathcal{G}_{X}$-bundle over $X$. This principal $\mathcal{G}_{X}$-bundle over $X$ will be denoted by $E_{\mathcal{G}_{X}}$.

Remark 2.4. A vector bundle $E$ over $X$ is called finite if there are two distinct polynomials $f_{1}, f_{2} \in \mathbb{Z}[t]$ with nonnegative coefficients such that the vector bundle $f_{1}(E)$ is isomorphic to $f_{2}(E)$. Any finite vector bundle over $X$ is strongly semistable of degree zero. A vector bundle $V$ of degree zero over $X$ is called essentially finite if there is a finite vector bundle $E$ over $X$ and a quotient bundle $Q$ of $E$ of degree zero such that the vector bundle $V$ is a subbundle of $Q$; see [6], [7]. Essentially finite vector bundles over $X$ form a neutral Tannakian category. and the corresponding group scheme is called the fundamental group scheme of $X$ [7]. Since any essentially finite vector bundle over $X$ is strongly semistable, the fundamental group scheme of $X$ is a quotient of the group scheme $\mathcal{G}_{X}$ constructed above.

\section{MONODROMY OF PRINCIPAL BUNDLES}

Let $G$ be a linear algebraic group defined over the field $k$. Let $Z_{0}^{\prime}(G)$ denote the connected component, containing the identity element, of the reduced center of $G$. We assume that $G$ satisfies the following condition: there is no nontrivial character of $G$ which is trivial on $Z_{0}^{\prime}(G)$. Let

$$
Z_{0}(G) \subset Z_{0}^{\prime}(G)
$$

be the (unique) maximal split torus contained in $Z_{0}^{\prime}(G)$. The above condition on $G$ implies that there is no nontrivial character of $G$ which is trivial on $Z_{0}(G)$.

The subgroup $Z_{0}(G)$ gives a decomposition of any $G$-module, which we will describe now.

Let $V$ be a finite dimensional left $G$-module. Consider the action on $V$ of the subgroup $Z_{0}(G)$ in eqn. (3.1). Since $Z_{0}(G)$ is a product of copies of $\mathbb{G}_{m}$, the vector space $V$ decomposes into a direct sum of one dimensional subspaces such that each of the one dimensional subspaces is preserved by the action of $Z_{0}(G)$. Therefore, we obtain a finite collection of distinct characters of $Z_{0}(G)$, say $\chi_{1}, \cdots, \chi_{m}$, such that for each line $\xi \subset V$ preserved by the action of $Z_{0}(G)$ on $V$, there is a character $\chi_{i}$, for some $i \in[1, m]$, such that $Z_{0}(G)$ acts on $\xi$ as scalar multiplications through the character $\chi_{i}$.

For any $i \in[1, m]$, let

$$
V_{i} \subset V
$$


be the linear subspace on which $Z_{0}(G)$ acts as scalar multiplications through the character $\chi_{i}$. This subspace $V_{i}$ is preserved by the action of $G$ on $V$. Indeed, this follows immediately from the fact that the actions of $G$ and $Z_{0}(G)$ on $V$ commute. Therefore, we have a natural decomposition

$$
V=V_{1} \bigoplus \cdots \bigoplus V_{m}
$$

of the $G$-module $V$. This is clearly the isotypical decomposition of the $Z_{0}(G)$-module $V$.

Let $Z_{0}(G)^{*}$ denote the group of all characters of $Z_{0}(G)$. We may reformulate the decomposition in eqn. (3.2) in the following way. Any finite dimensional left $G$-module has a natural decomposition

$$
V=\bigoplus_{\chi \in Z_{0}(G)^{*}} V_{\chi}
$$

where $V_{\chi} \subset V$ is the subspace on which $Z_{0}(G)$ acts as scalar multiplications through the character $\chi$.

As before, let $X$ be a geometrically irreducible smooth projective curve defined over the field $k$.

Lemma 3.1. Take any character $\chi \in Z_{0}(G)^{*}$. Let $V$ and $W$ be two nonzero finite dimensional left $G$-modules such that $Z_{0}(G)$ acts on both $V$ and $W$ as scalar multiplications through the character $\chi$. Let $E_{G}$ be a principal $G$-bundle over $X$. Let $E_{V}$ (respectively, $\left.E_{W}\right)$ be the vector bundle over $X$ associated to the principal $G$-bundle $E_{G}$ for the $G-$ module $V$ (respectively, $W$ ). Then

$$
\mu\left(E_{V}\right)=\mu\left(E_{W}\right) .
$$

Proof. The $G$-module structures on $V$ and $W$ together induce a $G$-module structure on the vector space $\operatorname{Hom}(V, W)=V^{*} \otimes W$. The vector bundle over $X$ associated to $E_{G}$ for the $G$-module $\operatorname{Hom}(V, W)$ is evidently identified with the vector bundle $E_{V}^{*} \otimes E_{W}$.

Since $Z_{0}(G)$ acts on both $V$ and $W$ as scalar multiplications through the character $\chi$, the action of $Z_{0}(G)$ on the $G$-module $\operatorname{Hom}(V, W)$ is the trivial action. In particular, the action of $Z_{0}(G)$ on the one-dimensional $G$-module $\bigwedge^{\text {top }} \operatorname{Hom}(V, W)$ is the trivial action.

By our assumption on $G$, the group $G / Z_{0}(G)$ does not admit any nontrivial character. Therefore, from the observation that $Z_{0}(G)$ acts trivially on the one-dimensional $G$ module $\bigwedge^{\text {top }} \operatorname{Hom}(V, W)$ we conclude that the action of $G$ on $\bigwedge^{\text {top }} \operatorname{Hom}(V, W)$ is the trivial action. This immediately implies that the line bundle over $X$ associated to the principal $G$-bundle $E_{G}$ for the $G$-module $\bigwedge^{\text {top }} \operatorname{Hom}(V, W)$ is a trivial line bundle.

The line bundle over $X$ associated to the principal $G$-bundle $E_{G}$ for the $G$-module $\bigwedge^{\text {top }} \operatorname{Hom}_{k}(V, W)$ is clearly identified with $\bigwedge^{\text {top }}\left(E_{V}^{*} \otimes E_{W}\right)$. Since $\bigwedge^{\text {top }}\left(E_{V}^{*} \otimes E_{W}\right)$ is a trivial line bundle, we have

$$
\operatorname{degree}\left(\bigwedge^{\text {top }}\left(E_{V}^{*} \bigotimes E_{W}\right)\right)=0
$$

Now from the identity

$$
\operatorname{degree}\left(\bigwedge\left(E_{V}^{*} \bigotimes E_{W}\right)\right)=\operatorname{degree}\left(E_{W}\right) \operatorname{rank}\left(E_{V}\right)-\operatorname{degree}\left(E_{V}\right) \operatorname{rank}\left(E_{W}\right),
$$


it follows that $\mu\left(E_{V}\right)=\mu\left(E_{W}\right)$. This completes the proof of the lemma.

The following is a corollary of [3, page 139, Proposition 2.21].

Corollary 3.2. All characters of $Z_{0}(G)$ arise from the indecomposable representations of $G$. In other words, for any character $\chi$ of $Z_{0}(G)$, there is some nonzero finite dimensional indecomposable left $G$-module $V$ such that $Z_{0}(G)$ acts on $V$ as scalar multiplications through the character $\chi$.

If $E$ and $F$ are two vector bundles over $X$, then $\mu(E \otimes F)=\mu(E)+\mu(F)$. Therefore, Lemma 3.1 and Corollary 3.2 combine together to give the following corollary:

Corollary 3.3. Fix any principal $G$-bundle $E_{G}$ over $X$. Then there is a homomorphism to the additive group

$$
\delta_{E_{G}}: Z_{0}(G)^{*} \longrightarrow \mathbb{Q}
$$

that sends any character $\chi$ to $\frac{\operatorname{degree}\left(E_{V}\right)}{\operatorname{rank}\left(E_{V}\right)}$, where $V$ is a finite dimensional nonzero left $G-$ module on which $Z_{0}(G)$ acts as scalar multiplications through the character $\chi$, and $E_{V}$ is the vector bundle over $X$ associated to the principal $G$-bundle $E_{G}$ for the $G$-module $V$.

Definition 3.4. Let $G$ be any affine group scheme defined over $k$. A principal $G$-bundle $E_{G}$ over a geometrically irreducible smooth projective curve $X$ will be called strongly semistable if for any indecomposable finite dimensional left $G$-module $V \in G$-mod, the vector bundle over $X$ associated to $E_{G}$ for $V$ is strongly semistable.

See [9], [8] for the definition of a (strongly) semistable principal bundle with a reductive group as the structure group. We will show that the above definition coincides with the usual definition when $G$ is reductive. For that we will need the following theorem.

Theorem 3.5 (RR, Theorem 3.23). Let $H$ and $H^{\prime}$ be reductive linear algebraic groups defined over $k$ and

$$
\rho: H \longrightarrow H^{\prime}
$$

a homomorphism of algebraic groups such that $\rho\left(Z_{0}^{\prime}(H)\right) \subset Z_{0}^{\prime}\left(H^{\prime}\right)$, where $Z_{0}^{\prime}(H)$ (respectively, $\left.Z_{0}^{\prime}\left(H^{\prime}\right)\right)$ is the connected component, containing the identity element, of the reduced center of $H$ (respectively, $H^{\prime}$ ). Let $E_{H}$ be a strongly semistable principal $H$-bundle over $X$. Then the principal $H^{\prime}$-bundle $E_{H^{\prime}}:=E_{H}\left(H^{\prime}\right)$ over $X$, obtained by extending the structure group of $E_{H}$ using $\rho$, is also strongly semistable.

Definition 3.4 is justified by the following lemma.

Lemma 3.6. Let $H$ be a reductive linear algebraic group and $X$ a geometrically irreducible smooth projective curve defined over $k$. A principal $H$-bundle $E_{H}$ over $X$ is strongly semistable if and only if for every indecomposable $H$-module $V$, the vector bundle $E_{V}=$ $E_{H}(V)$ over $X$ associated to the principal $H$-bundle $E_{H}$ for $V$ is strongly semistable.

Proof. Let $E_{H}$ be a strongly semistable principal $H$-bundle over $X$. Take any indecomposable $H$-module $V$. Since $V$ is indecomposable, the group $Z_{0}^{\prime}(H)$ acts on $V$ as scalar multiplications, where $Z_{0}^{\prime}(H)$ as before is the connected component, containing the identity element, of the reduced center of $H$. Therefore, from Theorem 3.5 it follows that the associated vector bundle $E_{V}$ is strongly semistable. 
To prove the converse, let $E_{H}$ be principal $H$-bundle over $X$ such that for every indecomposable $H$-module $V$, the vector bundle over $X$ associated to the principal $H$-bundle $E_{H}$ for $V$ is strongly semistable.

Let $\operatorname{ad}\left(E_{H}\right)$ be the adjoint vector bundle for $E_{H}$. We recall that $\operatorname{ad}\left(E_{H}\right)$ is the vector bundle over $X$ associated to the principal $H$-bundle $E_{H}$ for the adjoint action of $H$ on its Lie algebra $\mathfrak{h}$. To prove that the principal $H$-bundle $E_{H}$ is strongly semistable, it suffices to show that the vector bundle $\operatorname{ad}\left(E_{H}\right)$ is strongly semistable. To see this, let

$$
E_{P} \subset\left(F_{X}^{r}\right)^{*} E_{H}
$$

be a reduction of structure group to a maximal parabolic subgroup $P \subset H$ that violates the semistability condition, or in other words, we have

$$
\operatorname{degree}\left(\left(\left(F_{X}^{r}\right)^{*} \operatorname{ad}\left(E_{H}\right)\right) / \operatorname{ad}\left(E_{P}\right)\right)<0
$$

(see [9], [8]). Consider the subbundle

$$
\operatorname{ad}\left(E_{P}\right) \subset \operatorname{ad}\left(\left(F_{X}^{r}\right)^{*} E_{H}\right)=\left(F_{X}^{r}\right)^{*} \operatorname{ad}\left(E_{H}\right) .
$$

Since degree $\left(\operatorname{ad}\left(E_{H}\right)\right)=0$ (as $\bigwedge^{\text {top }} \mathfrak{h}$ is a trivial $H$-module), from eqn. (3.4) it follows immediately that the subbundle $\operatorname{ad}\left(E_{P}\right)$ contradicts the semistability condition of the vector bundle $\left(F_{X}^{r}\right)^{*} \operatorname{ad}\left(E_{H}\right)$.

Consider the Lie algebra $\mathfrak{h}$ as a $H$-module using the adjoint action. Note that $Z_{0}^{\prime}(H)$ acts trivially on $\mathfrak{h}$. Let

$$
\mathfrak{h}=\bigoplus_{i=1}^{n} V_{i}
$$

be a decomposition of $\mathfrak{h}$ into a direct sum of indecomposable $H$-modules. Let $E_{V_{i}}$ denote the vector bundle over $X$ associated to the principal $H$-bundle $E_{H}$ for the $H$-module $V_{i}$. From eqn. (3.5) we have

$$
\operatorname{ad}\left(E_{H}\right)=\bigoplus_{i=1}^{n} E_{V_{i}} .
$$

From the given condition on $E_{H}$ we know that $E_{V_{i}}$ is strongly semistable for all $i \in[1, n]$.

As $Z_{0}^{\prime}(H)$ acts trivially on $\mathfrak{h}$, and $H / Z_{0}(H)$ being semisimple does not have any nontrivial character, the induced action of $H$ on the line $\bigwedge^{\text {top }} V_{i}$ is the trivial action. Therefore, $\bigwedge^{\text {top }} E_{V_{i}}$ is a trivial line bundle. In particular,

$$
\operatorname{degree}\left(E_{V_{i}}\right)=0
$$

for all $i \in[1, n]$.

Since $\operatorname{ad}\left(E_{H}\right)$ is a direct sum of strongly semistable vector bundles of degree zero, we conclude that the vector bundle $\operatorname{ad}\left(E_{H}\right)$ is strongly semistable. This completes the proof of the lemma.

Let $E_{G}$ be a strongly semistable principal $G$-bundle over $X$. To each $G$-module we will associate an object of the neutral Tannakian category $\mathcal{C}_{X}$ that we constructed in Section 2 . 
Let $V$ be a finite dimensional left $G$-module. First consider the natural decomposition into a direct sum of $G$-modules

$$
V=\bigoplus_{\chi \in Z_{0}(G)^{*}} V_{\chi}
$$

constructed in eqn. (3.3). Let $E_{V_{\chi}}$ be the vector bundle over $X$ associated to the principal $G$-bundle $E_{G}$ for the above $G$-module $V_{\chi}$.

Lemma 3.7. The vector bundle $E_{V_{\chi}}$ is strongly semistable, and if $V_{\chi} \neq 0$, then

$$
\mu\left(E_{V_{\chi}}\right)=\delta_{E_{G}}(\chi),
$$

where $\delta_{E_{G}}$ is the homomorphism constructed in Corollary 3.3.

Proof. Expressing $V_{\chi}$ as a direct sum of indecomposable $G$-modules, and using the fact that a direct sum of strongly semistable vector bundles of same slope remains strongly semistable, we conclude that the associated vector bundle $E_{V_{\chi}}$ is strongly semistable (see Definition 3.4).

From the definition of $\delta_{E_{G}}$ it follows immediately that $\mu\left(E_{V_{\chi}}\right)=\delta_{E_{G}}(\chi)$ if $V_{\chi} \neq 0$. This completes the proof of the lemma.

Lemma 3.7 has the following corollary:

Corollary 3.8. For any $\lambda \in \mathbb{Q}$ and any $V \in G$-mod, the direct sum

$$
E_{G}^{\lambda}(V):=\bigoplus_{\left\{\chi \in Z_{0}(G)^{*} \mid \delta_{E_{G}}(\chi)=\lambda\right\}} E_{V_{\chi}}
$$

is either zero, or it is a strongly semistable vector bundle with

$$
\mu\left(E_{G}^{\lambda}(V)\right)=\lambda .
$$

Finally, consider the function $f_{E_{G}, V}$ from $\mathbb{Q}$ to the space of all vector bundles over $X$ defined by

$$
f_{E_{G}, V}(\lambda):=E_{G}^{\lambda}(V),
$$

where $V \in G$-mod, and $E_{G}^{\lambda}(V)$ is defined in Corollary 3.8. From Corollary 3.8 it follows immediately that this function $f_{E_{G}, V}$ is an object of the category $\mathcal{C}_{X}$ constructed in Section 2. Therefore, to each object of $G-\bmod$ we have associated an object of $\mathcal{C}_{X}$.

Our aim is to construct a Tannakian category out of the strongly semistable principal $G$-bundle $E_{G}$. Before that we will introduce some definitions.

Take any object $f$ in the category $\mathcal{C}_{X}$. A sub-object of $f$ is an object $f^{\prime}$ in $\mathcal{C}_{X}$ such that for each $\lambda \in \mathbb{Q}$, the vector bundle $f^{\prime}(\lambda)$ is a subbundle of the vector bundle $f(\lambda)$. If $f^{\prime}$ is a sub-object of $f$, then the object of $\mathcal{C}_{X}$ that sends any $\lambda \in \mathbb{Q}$ to the quotient vector bundle $f(\lambda) / f^{\prime}(\lambda)$ will be called a quotient-object of $f$.

Let $E$ be a strongly semistable vector bundle over $X$ and $E^{\prime}$ a nonzero proper subbundle of $E$ with

$$
\mu\left(E^{\prime}\right)=\mu(E) .
$$

Then $E^{\prime}$ is strongly semistable, and furthermore, the quotient vector bundle $E / E^{\prime}$ is also strongly semistable with $\mu\left(E / E^{\prime}\right)=\mu(E)$ if $E / E^{\prime} \neq 0$. Therefore, for an object $f$ of the 
category $\mathcal{C}_{X}$, any quotient-object of $f$ also lie in $\mathcal{C}_{X}$. Also, given a subbundle $V_{\lambda}^{\prime} \subset f(\lambda)$ for each $\lambda \in \mathbb{Q}$, to check that the function

$$
\lambda \longmapsto V_{\lambda}^{\prime}
$$

is a sub-object of $f$, all we need to check that

$$
\mu\left(V_{\lambda}^{\prime}\right)=\lambda
$$

for all $\lambda \in \mathbb{Q}$ with $V_{\lambda}^{\prime} \neq 0$.

For any object $f$ of the category $\mathcal{C}_{X}$, a sub-quotient of $f$ is a sub-object of some quotient-object of $f$.

Let $\mathcal{C}_{E_{G}}$ denote the subcategory of $\mathcal{C}_{X}$ defined by all objects $f$ of $\mathcal{C}_{X}$ such that there exists some $V \in G$-mod with the property that $f$ is isomorphic to a sub-quotient of $f_{E_{G}, V}$, where $f_{E_{G}, V}$ is the object of $\mathcal{C}_{X}$ constructed from $V$ in eqn. (3.6). The morphisms remain unchanged. In other words, for any two objects $f$ and $f^{\prime}$ in $\mathcal{C}_{E_{G}}$, the morphisms from $f$ to $f^{\prime}$ are the morphisms from $f$ to $f^{\prime}$ considered as objects of $\mathcal{C}_{X}$.

It is straight-forward to check that $\mathcal{C}_{E_{G}}$ is a neutral Tannakian subcategory of $\mathcal{C}_{X}$. Therefore, the neutral Tannakian category $\mathcal{C}_{E_{G}}$ gives an affine group scheme defined over $k$.

Definition 3.9. The affine group scheme defined over $k$ given by the neutral Tannakian category $\mathcal{C}_{E_{G}}$ will be called the monodromy group scheme of $E_{G}$. The monodromy group scheme of $E_{G}$ will be denoted by $M$.

Since $\mathcal{C}_{E_{G}}$ is a Tannakian subcategory of $\mathcal{C}_{X}$, the monodromy group scheme $M$ is a quotient of the group scheme $\mathcal{G}_{X}$ constructed in Section 2 (see [3, Proposition 2.21]). Let

$$
\phi_{E_{G}}: \mathcal{G}_{X} \longrightarrow M
$$

be the quotient map.

Just as we have the tautological $\mathcal{G}_{X}$-bundle $E_{\mathcal{G}_{X}}$ (see eqn. (2.7)), there is a tautological principal $M$-bundle over $X$.

Definition 3.10. Let $E_{M}$ denote the tautological principal $M$-bundle over $X$. This principal $M$-bundle $E_{M}$ will be called the monodromy bundle for $E_{G}$.

The principal $M$-bundle $E_{M}$ is evidently the one obtained by extending the structure group of the principal $\mathcal{G}_{X}$-bundle $E_{\mathcal{G}_{X}}$ using the homomorphism $\phi_{E_{G}}$ in eqn. (3.7).

We will next show that there is a tautological embedding of the monodromy group scheme $M$ into the fiber, over the fixed $k$-rational point $x \in X$, of the adjoint bundle for $E_{G}$.

Let $\operatorname{Ad}\left(E_{G}\right)$ be the adjoint bundle for the principal $G$-bundle $E_{G}$ over $X$. Let $\operatorname{Ad}\left(E_{G}\right)_{x}$ be the fiber of $\operatorname{Ad}\left(E_{G}\right)$ over the fixed $k$-rational point $x$ of $X$.

If $\omega$ is the fiber functor for the principal $G$-bundle $E_{G}$ over $X$, then the group $\operatorname{Ad}\left(E_{G}\right)_{x}$ defined over $k$ represents the functor $\underline{\mathrm{Aut}}^{\otimes}(\omega)$. Using Theorem 2.11 in [3], page 130], we get a natural homomorphism from the group scheme $\mathcal{G}_{X}$ (constructed in Section 2) to $\operatorname{Ad}\left(E_{G}\right)_{x}$. Let

$$
\Phi\left(E_{G}\right): \mathcal{G}_{X} \longrightarrow \operatorname{Ad}\left(E_{G}\right)_{x}
$$


be this natural homomorphism.

It is easy to see that the image of the homomorphism $\Phi\left(E_{G}\right)$ in eqn. (3.8) coincides with the monodromy group scheme $M$ in Definition 3.9.

Therefore, we have the following proposition:

Proposition 3.11. The monodromy group scheme $M$ for $E_{G}$ (introduced in Definition [3.9) is identified with the image of the homomorphism $\Phi\left(E_{G}\right)$ in eqn. (3.8). In other words, the kernel of the homomorphism $\Phi\left(E_{G}\right)$ coincides with the kernel of the homomorphism $\phi_{E_{G}}$ in eqn. (3.7).

There is a natural inclusion $M \hookrightarrow \operatorname{Ad}\left(E_{G}\right)_{x}$ obtained from the fact that the quotients of $\mathcal{G}_{X}$ for the two homomorphisms $\Phi\left(E_{G}\right)$ and $\phi_{E_{G}}$ coincide.

We will now investigate the behavior of the monodromy group and the monodromy bundle under the extensions of structure group.

Let

$$
\rho: G \longrightarrow G_{1}
$$

be an algebraic homomorphism between linear algebraic groups defined over $k$. Let $Z_{0}\left(G_{1}\right)$ denote the (unique) maximal split torus contained in the reduced center of $G_{1}$. We assume the following:

- The group $G_{1}$ does not admit any nontrivial character which is trivial on $Z_{0}\left(G_{1}\right)$.

- The homomorphism $\rho$ in eqn. (3.9) satisfies the condition

$$
\rho\left(Z_{0}(G)\right) \subset Z_{0}\left(G_{1}\right) .
$$

Lemma 3.12. Let $E_{G}$ be a strongly semistable principal $G$-bundle over $X$. Then the principal $G_{1}$-bundle $E_{G_{1}}:=E_{G}\left(G_{1}\right)$, obtained by extending the structure group of $E_{G}$ using $\rho$ (defined in eqn. (3.9) ) is also strongly semistable.

Proof. Take an indecomposable $G_{1}$-module $V$. So $Z_{0}\left(G_{1}\right)$ acts on $V$ as scalar multiplications through a character. Therefore, using eqn. (3.10) we know that $Z_{0}(G)$ acts on $V$ as scalar multiplications through a character. Since $E_{G}$ is strongly semistable, Corollary 3.8 says that the associated vector bundle $E_{G}(V)=E_{G_{1}}(V)$ is strongly semistable. Hence $E_{G_{1}}$ is strongly semistable (see Definition 3.4).

Let $E_{G}$ be a strongly semistable principal $G$-bundle over $X$. Hence by Lemma 3.12, the principal $G_{1}$-bundle $E_{G_{1}}$, obtained by extending the structure group of the principal $G^{-}$ bundle $E_{G}$ using the homomorphism $\rho$, is also strongly semistable. The homomorphism $\rho$ in eqn. (3.9) induces a homomorphism of group schemes

$$
\widetilde{\rho}: \operatorname{Ad}\left(E_{G}\right) \longrightarrow \operatorname{Ad}\left(E_{G_{1}}\right)
$$

over $X$.

Lemma 3.13. The monodromy group scheme $M_{1} \subset \operatorname{Ad}\left(E_{G_{1}}\right)_{x}$ for $E_{G_{1}}$ is the image $\widetilde{\rho}(x)(M)$, where $\widetilde{\rho}(x)$ is the homomorphism in eqn. (3.11) restricted to the $k$-rational point $x$ of $X$, and $M \subset \operatorname{Ad}\left(E_{G}\right)_{x}$ is the monodromy group scheme of $E_{G}$ (see Proposition 3.11). Furthermore, the monodromy bundle $E_{M_{1}}$ for $E_{G_{1}}$ is the extension of structure 
group of the monodromy bundle $E_{M}$ for $E_{G}$ by the homomorphism $M \longrightarrow M_{1}$ obtained by restricting $\widetilde{\rho}(x)$.

Proof. Take any $G_{1}-$ module $V \in G_{1}-$ mod. The $G$-module given by $V$ using the homomorphism $\rho$ in eqn. (3.9) will also be denoted by $V$. Consider the isotypical decomposition of the $Z_{0}(G)$-module $V$, and also consider the isotypical decomposition of the $Z_{0}\left(G_{1}\right)$ module $V$. The second decomposition is finer in the following sense. From eqn. (3.10) we get a homomorphism

$$
\rho^{*}: Z_{0}\left(G_{1}\right)^{*} \longrightarrow Z_{0}(G)^{*}
$$

of character groups. For any $\chi \in Z_{0}(G)^{*}$, the component of $V$ on which $Z_{0}(G)$ acts as scalar multiplications through $\chi$ is the direct sum

$$
\bigoplus_{\chi^{\prime} \in\left(\rho^{*}\right)^{-1}(\chi)} V_{\chi^{\prime}}
$$

where $V_{\chi^{\prime}} \subset V$ is the subspace on which $Z_{0}\left(G_{1}\right)$ acts as scalar multiplications through $\chi^{\prime}$.

Using this observation it follows that the neutral Tannakian category $\mathcal{C}_{E_{G_{1}}}$ for the principal $G_{1}$-bundle $E_{G_{1}}$ is a subcategory of the neutral Tannakian category $\mathcal{C}_{E_{G}}$ for $E_{G}$. Now the lemma follows from the constructions of the monodromy group scheme and the monodromy bundle and the criterion for surjectivity in [3, page 139, Proposition $2.21(\mathrm{a})]$.

\section{Some Properties of THE MONODROMY GROUP SCHEME}

As before, let $G$ be a linear algebraic group defined over $k$ which does not admit any nontrivial character trivial on $Z_{0}(G)$. Take a principal $G$-bundle $E_{G}$ over the curve $X$.

For a subgroup scheme $H \subset G$, let $\left(H \cap Z_{0}(G)\right)_{\text {red }}$ denote the reduced intersection; let $\left(H \bigcap Z_{0}(G)\right)_{0}$ denote the (unique) maximal split torus contained in the abelian group $\left(H \cap Z_{0}(G)\right)_{\text {red }}$.

Definition 4.1. A reduction of structure group

$$
E_{H} \subset E_{G}
$$

of $E_{G}$ to a subgroup scheme $H \subset G$ will be called balanced if for every character $\chi$ of $H$ trivial on $\left(H \cap Z_{0}(G)\right)_{0} \subset H$ (see the above definition), we have

$$
\text { degree }\left(E_{H}(\chi)\right)=0 \text {, }
$$

where $E_{H}(\chi)$ is the line bundle over $X$ associated to the principal $H$-bundle $E_{H}$ for the character $\chi$.

Remark 4.2. Since any character of $\left(H \bigcap Z_{0}(G)\right)_{\text {red }} /\left(H \bigcap Z_{0}(G)\right)_{0}$ is of finite order, if a character $\chi$ of $H$ is trivial on $\left(H \cap Z_{0}(G)\right)_{0}$, then there is a positive integer $n$ such that the character $\chi^{n}$ of $H$ is trivial on $\left(H \bigcap Z_{0}(G)\right)_{\text {red }}$. Therefore, a reduction $E_{H} \subset E_{G}$ as in Definition 4.1 is balanced if and only if for every character $\chi$ of $H$ trivial on $\left(H \cap Z_{0}(G)\right)_{\text {red }}$ we have

$$
\operatorname{degree}\left(E_{H}(\chi)\right)=0 .
$$


Since the quotient $\left(H \bigcap Z_{0}(G)\right) /\left(H \bigcap Z_{0}(G)\right)_{\text {red }}$ is a finite group scheme, if a character $\chi$ of $H$ is trivial on $\left(H \cap Z_{0}(G)\right)_{\text {red }}$, then there is a positive integer $n$ such that the character $\chi^{n}$ of $H$ is trivial on $H \bigcap Z_{0}(G)$. Therefore, a reduction $E_{H} \subset E_{G}$ as in Definition 4.1 is balanced if and only if for every character $\chi$ of $H$ trivial on $H \bigcap Z_{0}(G)$ we have

$$
\operatorname{degree}\left(E_{H}(\chi)\right)=0 \text {. }
$$

Proposition 4.3. Let $E_{G}$ be a strongly semistable principal $G$-bundle over $X$ and $E_{H} \subset$ $E_{G}$ a balanced reduction of structure group of $E_{G}$ to a subgroup scheme $H \subset G$. Then the principal $H$-bundle $E_{H}$ over $X$ is strongly semistable.

Proof. Take any indecomposable $H$-module $W$. Let $E_{W}=E_{H}(W)$ be the vector bundle over $X$ associated to the principal $H$-bundle $E_{H}$ for the $H$-module $W$. We need to show that $E_{W}$ is strongly semistable. For that it suffices to show that the vector bundle $\operatorname{End}\left(E_{W}\right)$ is strongly semistable. Indeed, if a subbundle $F \subset\left(F_{X}^{j}\right)^{*} E_{W}$ contradicts the semistability condition of the vector bundle $\left(F_{X}^{j}\right)^{*} E_{W}$, then the subbundle

$$
\left(\left(F_{X}^{j}\right)^{*} E_{W} / F\right)^{*} \bigotimes\left(F_{X}^{j}\right)^{*} E_{W} \subset\left(\left(F_{X}^{j}\right)^{*} E_{W}\right)^{*} \bigotimes\left(F_{X}^{j}\right)^{*} E_{W}=\left(F_{X}^{j}\right)^{*} \operatorname{End}\left(E_{W}\right)
$$

contradicts the semistability condition of the vector bundle $\left(F_{X}^{j}\right)^{*} \operatorname{End}\left(E_{W}\right)$.

Let

$$
\rho: H \longrightarrow \mathrm{GL}(\operatorname{End}(W))
$$

be the homomorphism given by the action of $H$ on $\operatorname{End}(W)$ induced by the action of $H$ on $W$. We note that $\operatorname{End}\left(E_{W}\right)$ is the vector bundle associated to the principal $H$-bundle $E_{H}$ for the $H$-module $\operatorname{End}(W)$.

Since the $H$-module $W$ is indecomposable, the group scheme $H \cap Z_{0}(G)$ acts on $W$ as scalar multiplications through a character of $H \bigcap Z_{0}(G)$. Therefore, $H \bigcap Z_{0}(G)$ acts trivially on $\operatorname{End}(W)$. In other words, $\operatorname{End}(W)$ is an $H /\left(H \bigcap Z_{0}(G)\right)$-module.

As $H$ is a subgroup scheme of $G$, we have

$$
H /\left(H \bigcap Z_{0}(G)\right) \subset G / Z_{0}(G) .
$$

Therefore, there is a $G / Z_{0}(G)$-module $V$ such that the $H /\left(H \bigcap Z_{0}(G)\right)$-module $\operatorname{End}(W)$ is a subquotient of the $H /\left(H \bigcap Z_{0}(G)\right)$-module $V$ (see [3, page 139, Proposition 2.21(b)]). Let

$$
V \rightarrow Q
$$

be a quotient of the $H /\left(H \bigcap Z_{0}(G)\right)$-module $V$ such that $\operatorname{End}(W)$ is a submodule of the $H /\left(H \bigcap Z_{0}(G)\right)$-module $Q$.

Let

$$
V=\bigoplus_{i=1}^{\ell} V_{i}
$$

be a decomposition of the $G / Z_{0}(G)$-module $V$ into a direct sum of indecomposable $G / Z_{0}(G)$-modules. For any $i \in[1, \ell]$, let $E_{V_{i}}$ be the vector bundle over $X$ associated to the principal $G$-bundle $E_{G}$ for the $G$-module $V_{i}$ (the $G / Z_{0}(G)$-module $V_{i}$ is considered as a $G$-module using the quotient map to $G / Z_{0}(G)$ ). 
Since $E_{G}$ is strongly semistable, and the $G$-module $V_{i}$ is indecomposable, the associated vector bundle $E_{V_{i}}$ is strongly semistable. As $G / Z_{0}(G)$ does not admit any nontrivial characters, the induced action of $G$ on the line $\bigwedge^{\text {top }} V_{i}$ is the trivial action. Therefore, the associated line bundle $\bigwedge^{\text {top }} E_{V_{i}}$ is trivializable. In particular, we have

$$
\operatorname{degree}\left(E_{V_{i}}\right)=0 \text {. }
$$

Since each $E_{V_{i}}$ is strongly semistable of degree zero, the vector bundle $E_{V}$ is also strongly semistable of degree zero.

Let $E_{Q}$ denote the vector bundle over $X$ associated to the principal $H$-bundle $E_{H}$ for the $H$-module $Q$ in eqn. (4.3) (the $H /\left(H \bigcap Z_{0}(G)\right)$-module $Q$ is considered as an $H$ module using the quotient map to $\left.H /\left(H \bigcap Z_{0}(G)\right)\right)$. Since $H \bigcap Z_{0}(G)$ acts trivially on the line $\bigwedge^{\text {top }} Q$, and $E_{H} \subset E_{G}$ is a balanced reduction of structure group, we have

$$
\operatorname{degree}\left(E_{Q}\right)=\operatorname{degree}\left(E_{H}(\bigwedge Q)\right)=0
$$

where $E_{H}\left(\bigwedge^{\text {top }} Q\right)$ is the line bundle over $X$ associated to the principal $H$-bundle $E_{H}$ for the $H$-module $\bigwedge^{\text {top }} Q$.

Since $Q$ is a quotient of the $H$-module $V$, the vector bundle $E_{Q}$ is a quotient of $E_{V}$. The vector bundle $E_{V}$ is strongly semistable of degree zero, and $E_{Q}$ is a quotient of it of degree zero. Hence the vector bundle $E_{Q}$ is also strongly semistable.

We recall that the $H$-module $\operatorname{End}(W)$ is a submodule of the $H$-module $Q$. Therefore, the associated vector bundle $\operatorname{End}\left(E_{W}\right)$ is a subbundle of $E_{Q}$. Since $E_{Q}$ is a strongly semistable vector bundle of degree zero, and $\operatorname{End}\left(E_{W}\right)$ is a subbundle of it of degree zero, we conclude that the vector bundle $\operatorname{End}\left(E_{W}\right)$ is strongly semistable.

We saw earlier that $E_{W}$ is strongly semistable if $\operatorname{End}\left(E_{W}\right)$ is so. Therefore, the principal $H$-bundle $E_{H}$ is strongly semistable. This completes the proof of the proposition.

Let $E_{G}$ be a strongly semistable principal $G$-bundle over $X$. In Proposition 3.11 we saw that the monodromy group scheme $M$ (constructed in Definition 3.9) is canonically embedded in $\operatorname{Ad}\left(E_{G}\right)_{x}$. For national convenience, we will denote by $\widetilde{G}$ the group $\operatorname{Ad}\left(E_{G}\right)_{x}$ defined over $k$. Let $E_{\widetilde{G}}$ be the principal $\widetilde{G}$-bundle over $X$ obtained by extending the structure group of the monodromy bundle $E_{M}$ (see Definition 3.10) using the inclusion of $M$ in $\widetilde{G}$. Therefore,

$$
E_{M} \subset E_{\widetilde{G}}
$$

is a reduction of structure group of $E_{\widetilde{G}}$ to $M$.

Let $Z_{0}(\widetilde{G})$ denote the unique maximal split torus contained in the reduced center of $\widetilde{G}$.

Theorem 4.4. Assume that the group $\widetilde{G}:=A d\left(E_{G}\right)_{x}$ does not admit any nontrivial character which is trivial on $Z_{0}(\widetilde{G})$. Then the reduction of structure group in eqn. (4.5) is a balanced reduction of structure group of $E_{\widetilde{G}}$ to $M$. In particular, the principal $M$-bundle $E_{M}$ is strongly semistable.

Proof. We first note that the quotient $M /\left(M \bigcap Z_{0}(\widetilde{G})\right)$ is a subgroup scheme of

$$
G^{\prime}:=\widetilde{G} / Z_{0}(\widetilde{G}) \text {. }
$$


Therefore, any $M /\left(M \cap Z_{0}(\widetilde{G})\right)$-module is a subquotient of some $G^{\prime}$-module considered as a $M /\left(M \cap Z_{0}(G)\right)$-module (see [3, page 139, Proposition 2.21(b)]). Let $\chi$ be a character of $M$ which is trivial on the group scheme $M \bigcap Z_{0}(\widetilde{G})$. Let $L$ denote the line bundle over $X$ associated to the principal $M$-bundle $E_{M}$ for the character $\chi$. To prove that $E_{M} \subset E_{\widetilde{G}}$ is a balanced reductive of structure group, it suffices to show that degree $(L)=0$ (see Remark 4.2).

The one-dimensional $M$-module corresponding to $\chi$ will be denoted by $\xi$. Since $\chi$ is trivial on $M \bigcap Z_{0}(\widetilde{G})$, the $M$-module $\xi$ is given by a $M /\left(M \bigcap Z_{0}(\widetilde{G})\right)$-module. This $M /\left(M \cap Z_{0}(\widetilde{G})\right)$-module will also be denoted by $\xi$. Let $V$ be a $G^{\prime}$-module such that the $M /\left(M \cap Z_{0}(\widetilde{G})\right)$-module $\xi$ is a subquotient of $V$ (we noted earlier that any $M /\left(M \bigcap Z_{0}(\widetilde{G})\right)$-module is a subquotient of some $G^{\prime}$-module).

Since $E_{G}$ is strongly semistable, the $\widetilde{G}$-bundle $E_{\widetilde{G}}$ is also strongly semistable. Let $E_{V}$ denote the vector bundle over $X$ associated to the principal $\widetilde{G}$-bundle $E_{\widetilde{G}}$ for the $\widetilde{G}$ module $V$ (since $G^{\prime}$ is a quotient of $\widetilde{G}$, any $G^{\prime}$-module is also a $\widetilde{G}$-module). As $Z_{0}(\widetilde{G})$ acts trivially on $V$, and the principal $\widetilde{G}$-bundle $E_{\widetilde{G}}$ is strongly semistable, the associated vector bundle $E_{V}$ is also strongly semistable (see Lemma 3.12).

By our assumption, $G^{\prime}$ does not admit any nontrivial character. Therefore, the onedimensional $\widetilde{G}$-module $\bigwedge^{\text {top }} V$ is a trivial $\widetilde{G}$-module. Consequently, the line bundle over $X$ associated to the principal $\widetilde{G}$-bundle $E_{\widetilde{G}}$ for the one-dimensional $\widetilde{G}$-module $\bigwedge^{\text {top }} V$ is a trivializable. Hence, we have

$$
\operatorname{degree}\left(E_{V}\right)=\operatorname{degree}\left(\bigwedge^{\text {top }} E_{V}\right)=0
$$

The earlier defined line bundle $L$ over $X$ is the one associated to the principal $M$-bundle $E_{M}$ for the $M$-module $\xi$. From the definition of the monodromy bundle $E_{M}$ it follows immediately that $L$ is an object of the category $\mathcal{C}_{X}$ (defined in Section 2).

We recall that the $M$-module $\xi$ is a subquotient of the $M$-module $V$. This means that the object $L$ of $\mathcal{C}_{X}$ is a subquotient of the object $E_{V}$ of $\mathcal{C}_{X}$. On the other hand, $E_{V}$ is a strongly semistable vector bundle of degree zero. Therefore, we conclude that

$$
\operatorname{degree}(L)=0 \text {. }
$$

Thus, $E_{M} \subset E_{\widetilde{G}}$ is a balanced reduction of structure group of $E_{\widetilde{G}}$ to $M$. Now from Proposition 4.3 it follows that the principal $M$-bundle $E_{M}$ is strongly semistable. This completes the proof of the theorem.

Remark 4.5. Assume that the fiber of the principal bundle $E_{G}$, over the $k$-rational point $x$ of $X$, admits a rational point. If we fix a rational point in the fiber of $E_{G}$ over $x$, then $\widetilde{G}$ gets identified with $G$, and the principal bundle $E_{\widetilde{G}}$ gets identified with $E_{G}$.

If $E_{H} \subset E_{G}$ is a reduction of structure group, to a subgroup scheme $H \subset G$, of a principal $G$-bundle $E_{G}$ over $X$, then the adjoint bundle $\operatorname{Ad}\left(E_{H}\right)$ is a subgroup scheme of the group scheme $\operatorname{Ad}\left(E_{G}\right)$ over $X$.

Theorem 4.6. Let $E_{G}$ be a strongly semistable principal $G$-bundle over a geometrically irreducible smooth projective curve $X$ defined over $k$, where $G$ is a linear algebraic group 
defined over $k$ with the property that $G$ does not admit any nontrivial character which is trivial on $Z_{0}(G)$. Fix a k-rational point $x$ of $X$. Let $H \subset G$ be a subgroup scheme and $E_{H} \subset E_{G}$ a balanced reduction of structure group of $E_{G}$ to $H$. Then the image in $\operatorname{Ad}\left(E_{G}\right)_{x}$ of the monodromy group scheme $M$ (image by the homomorphism in Proposition 3.11) is contained in the subgroup scheme $\operatorname{Ad}\left(E_{H}\right)_{x} \subset \operatorname{Ad}\left(E_{G}\right)_{x}$.

Proof. Take any indecomposable $H$-module $V$. Let $\chi$ be the character of $\left(H \bigcap Z_{0}(G)\right)_{0}$ corresponding to the indecomposable $H$-module $V$ (see Definition 4.1). Let $E_{V}$ denote the vector bundle over $X$ associated to the principal $H$-bundle $E_{H}$ for the $H$-module $V$. To prove the theorem it suffices to show that $E_{V}$ is strongly semistable, and it is an object of the neutral Tannakian category $\mathcal{C}_{E_{G}}$. (We recall that the monodromy group $M$ is constructed from $\mathcal{C}_{E_{G}}$; see Definition 3.9.)

Since $E_{H} \subset E_{G}$ is a balanced reduction of structure group of $E_{G}$ to $H$, from Proposition 4.3 we know that the principal $H$-bundle $E_{H}$ is strongly semistable. As the $H$-module $V$ is indecomposable, and the principal $H$-bundle $E_{H}$ is strongly semistable, we conclude that the associated vector bundle $E_{V}$ is strongly semistable. Therefore, to complete the proof of the theorem we need to show that $E_{V}$ is an object of the neutral Tannakian category $\mathcal{C}_{E_{G}}$.

We recall that the group $\left(Z_{0}(G) \cap H\right)_{0}$ is a product of copies of the multiplicative group $\mathbb{G}_{m}$. Therefore, the inclusion

$$
\left(Z_{0}(G) \bigcap H\right)_{0} \hookrightarrow Z_{0}(G)
$$

splits [2, §8.5, page 115, Corollary]. Also, in Lemma 3.2 we showed that any character of $Z_{0}(H)$ arises from an indecomposable $G$-module. Therefore, there is an indecomposable $G$-module $\widehat{V}$ such that $\left(Z_{0}(G) \bigcap H\right)_{0}$ acts on $\widehat{V}$ as scalar multiplication through the earlier defined character $\chi$ (the character through which $\left(Z_{0}(G) \cap H\right)_{0}$ acts on $V$ ).

Let $E_{\widehat{V}}$ be the vector bundle over $X$ associated to the principal $G$-bundle $E_{G}$ for the $G$-module $\widehat{V}$. Since the $G$-module $\widehat{V}$ is indecomposable, the subgroup $Z_{0}(G)$ acts on $\widehat{V}$ as scalar multiplications. As the principal $G$-bundle $E_{G}$ is strongly semistable, this implies that the associated vector bundle $E_{\widehat{V}}$ is strongly semistable (see Corollary 3.8).

Since both the vector bundles $E_{V}$ and $E_{\widehat{V}}$ are strongly semistable, the vector bundle

$$
W:=E_{\widehat{V}}^{*} \bigotimes E_{V}
$$

is also strongly semistable (see Theorem 3.5).

As both $E_{\widehat{V}}$ and $W$ are strongly semistable, the vector bundle $E_{\widehat{V}} \otimes W$ is also strongly semistable (see Theorem 3.5). Furthermore, the vector bundle $E_{V}$ is a subbundle of $E_{\widehat{V}} \otimes W=\operatorname{End}\left(E_{\widehat{V}}\right) \otimes E_{V}$; note that $\mathcal{O}_{X}$ is subbundle of $\operatorname{End}\left(E_{\widehat{V}}\right)$, and hence $E_{V}$ is a subbundle of $\operatorname{End}\left(E_{\widehat{V}}\right) \otimes E_{V}$. We also have

$$
\mu\left(E_{\widehat{V}} \bigotimes W\right)=\mu\left(E_{V}\right)
$$

Therefore, to show that the strongly semistable vector bundle $E_{V}$ is an object of the Tannakian category $\mathcal{C}_{E_{G}}$, it suffices to show that the strongly semistable vector bundle $W$ is an object of the neutral Tannakian category $\mathcal{C}_{E_{G}}$. 
The group $\left(Z_{0}(G) \bigcap H\right)_{0}$ acts on both $\widehat{V}$ and $V$ as multiplication by scalars through the character $\chi$. This immediately implies that $\left(Z_{0}(G) \cap H\right)_{0}$ acts trivially on the $H$-module $\widehat{V}^{*} \otimes V$. Set

$$
H^{\prime}:=H /\left(Z_{0}(G) \bigcap H\right)_{0}
$$

Therefore, $\widehat{V}^{*} \otimes V$ is an $H^{\prime}$-module.

Set

$$
G^{\prime}:=G /\left(Z_{0}(G) \bigcap H\right)_{0}
$$

Since $H^{\prime}$ is a subgroup scheme of $G^{\prime}$, there is a $G^{\prime}$-module $V^{\prime}$ such that the $H^{\prime}$-module $\widehat{V}^{*} \otimes V$ is a subquotient of $V^{\prime}$ considered as an $H^{\prime}$-module (see [3, page 139, Proposition $2.21(\mathrm{~b})])$.

Let

$$
V^{\prime} \rightarrow Q
$$

be a quotient of the $H^{\prime}$-module $V^{\prime}$ such that the $H^{\prime}$-module $\widehat{V}^{*} \otimes V$ is a submodule of $Q$.

Let $E_{V^{\prime}}$ be the vector bundle over $X$ associated to the principal $G$-bundle $E_{G}$ for the $G$-module $V^{\prime}$. We will show that $E_{V^{\prime}}$ is strongly semistable of degree zero.

For that, express the $H^{\prime}$-module $V^{\prime}$ as a direct sum of indecomposable $H^{\prime}$-modules. Since $E_{H} \subset E_{G}$ is a balanced reduction of structure group, the vector bundle over $X$ associated to the principal $H$-bundle $E_{H}$ for an indecomposable $H^{\prime}$-module is strongly semistable of degree zero; see Definition 4.1 and Proposition 4.3. Therefore, $E_{V^{\prime}}$ is isomorphic to a direct sum of strongly semistable vector bundles of degree zero (corresponding to a decomposition of $V^{\prime}$ as a direct sum of indecomposable $H^{\prime}$-modules). Hence $E_{V^{\prime}}$ is a strongly semistable vector bundle of degree zero. We also note that $E_{V^{\prime}}$ is an object of the Tannakian category $\mathcal{C}_{E_{G}}$.

Consider the $H^{\prime}$-module $Q$ in eqn. (4.7). Let $E_{Q}$ denote the vector bundle over $X$ associated to the principal $H$-bundle $E_{H}$ for the $H$-module $Q$. Since the $H$-module $Q$ is a quotient of $V^{\prime}$, the vector bundle $E_{Q}$ is a quotient bundle of $E_{V^{\prime}}$. As the reduction $E_{H} \subset E_{G}$ is balanced, we have $\operatorname{degree}\left(E_{Q}\right)=0$ (recall that $Q$ is an $H^{\prime}$-module). Since $E_{Q}$ is a quotient bundle of degree zero of the strongly semistable vector bundle $E_{V^{\prime}}$ of degree zero, we conclude that $E_{Q}$ is strongly semistable. Therefore, the vector bundle $E_{Q}$ is also an object of the Tannakian category $\mathcal{C}_{E_{G}}$.

Finally, the vector bundle $W$ (defined in eqn. (4.6)) is a subbundle of $E_{Q}$, because the $H^{\prime}$-module $\widehat{V}^{*} \otimes V$ is submodule of $Q$. Since the reduction $E_{H} \subset E_{G}$ is balanced, and $\widehat{V}^{*} \otimes V$ is an $H^{\prime}$-module, we conclude that $\operatorname{degree}(W)=0$. As $E_{Q}$ is an object of the Tannakian category $\mathcal{C}_{E_{G}}$, and $E_{Q}$ is strongly semistable of degree zero, we conclude that the subbundle $W \subset E_{Q}$ of degree zero is strongly semistable, and furthermore, $W$ is an object of $\mathcal{C}_{E_{G}}$. This completes this proof of the theorem.

Acknowledgements. The referee gave detailed comments how the results (of a previous version) could be generalized. The referee also gave suggestions to improve the exposition. We are very grateful to the referee. 


\section{REFERENCES}

[1] Biswas, I., Parameswaran, A. J. and Subramanian, S.: Monodromy group for a strongly semistable principal bundle over a curve, Duke Math. Jour. 132 (2006), 1-48.

[2] Borel, A.: Linear Algebraic Groups, Second edition. Graduate Texts in Mathematics, 126. Springer-Verlag, New York, 1991.

[3] Deligne, P. and Milne, J. S.: Tannakian Categories, in: Hodge cycles, motives, and Shimura varieties (by P. Deligne, J. S. Milne, A. Ogus and K.-Y. Shih), pp. 101-228, Lecture Notes in Mathematics, 900, Springer-Verlag, Berlin-Heidelberg-New York, 1982.

[4] Harder, G. and Narasimhan, M. S.: On the cohomology groups of moduli spaces of vector bundles on curves, Math. Ann. 212 (1975), 215-248.

[5] Langton, S.G.: Valuative criteria for families of vector bundles on algebraic varieties, Ann. of Math. 101 (1975), 88-110.

[6] Nori, M. V.: On the representations of the fundamental group scheme, Compos. Math. 33 (1976), 29-41.

[7] Nori, M. V.: The fundamental group scheme, Proc. Ind. Acad. Sci. (Math. Sci.) 91 (1982), $73-122$.

[8] Ramanan, S. and Ramanathan, A.: Some remarks on the instability flag, Tôhoku Math. Jour. 36 (1984), 269-291.

[9] Ramanathan, A.: Stable principal bundles on a compact Riemann surface, Math. Ann. 213 (1975), 129-152.

[10] Saavedra Rivano, N.: Catégories Tannakiennes, Lecture Notes in Mathematics, 265, SpringerVerlag, Berlin-Heidelberg-New York, 1972.

School of Mathematics, Tata Institute of Fundamental Research, Homi Bhabha Road, Mumbai 400005, India

E-mail address: indranil@math.tifr.res.in

School of Mathematics, Tata Institute of Fundamental Research, Homi Bhabha Road, Mumbai 400005, INDiA

E-mail address: param@math.tifr.res.in 